\title{
Maximal Nucleus Clusters in Pawlak Paintings. Nerves as approximating tools in Visual Arts
}

\author{
James Peters \\ Dept Electrical \& Computer Engineering \\ University of Manitoba \\ Winnipeg, Manitoba R3T 5V6, CANADA \\ Email: james.peters3@umanitoba.ca
}

\author{
Sheela Ramanna \\ Dept. of Applied Computer Science \\ University of Winnipeg \\ Winnipeg, Manitoba R3B 2E9, CANADA \\ Email: s.ramanna@uwinnipeg.ca
}

\begin{abstract}
This paper is an application of Edelsbrunner-Harer (EH) nerves as approximating tools in discovering interesting perceptual clusters in Pawlak's painting of landscapes, thus giving us an insight into the style of the artist. A variation of EH nerves (collections of Voronoï regions called nucleus clusters) are used in this paper. The Rényi entropy is used to measure the information level of Voronoï regions. It is shown that the information levels (i.e., Rényi entropy) of maximal nucleus clusters in tesselled paintings are the highest compared with surrounding regions, thereby highlighting regions in the paintings with the greatest detail by the artist.
\end{abstract}

\section{INTRODUCTION}

$\mathbf{I}$ $\mathrm{N}$ THIS paper, we are seeking to discover interesting clusters using the concept of nerves useful in visual arts. Visual art forms include paintings, ceramics, photography amongst others. Both spatial and descriptive forms of representation will be considered [1]. First, we start by considering a representative space for the visual information in paintings by Z. Pawlak. Spatial representation can be considered in two ways: i) one where a space is given and its characteristics are studied via geometry and topology ii) a space is approximated using some form of tool [2]. Descriptive representation starts with probe functions that map features of objects to numbers in $\mathbb{R}^{n}$ [1]. Probe values provide a description of an object. The problem of finding interesting clusters in an object space $X$ is mapped to the problem of finding interesting clusters in a feature space $\Phi(X)$. The nearness of feature space clusters is studied in the context of proximity spaces.

Various forms of geometric nerves are usually collections known as simplicial complexes in a normed linear space (for details see, e.g. , [3], [1, §1.13]). A nerve $N(C)$ in a finite collection of sets $C$ is a simplicial complex with vertices of sets in $C$ and with simplices corresponding to all non-empty intersections among these sets.

In a descriptive representation, the simplicial complexes are a result of nerve constructions of observations (objects) in the feature space. To construct the simplicial complexes, we tessellate Pawlak Paintings with Voronoi diagram overlays. Then we compute nerves of sets of collections derived from these Voronoi regions [4]. A variation of Edelsbrunner-Harer nerves which are collections of Voronoï regions (called nucleus clusters) are used in this paper. Rényi entropy is used to measure the information level of Voronoï tessellation cells [5].
The focus here is on maximal nucleus clusters (MNCs) that are strongly proximal Edelsbrunner-Harer nerves. A proximity space setting for MNCs makes it possible to investigate the strong closeness of subsets in MNCs as well as the spatial and descriptive closeness of MNCs themselves.

Voronoi tessellation has great utility and has many applications such as geometric modelling in physics, astrophysics, chemistry and biology [6] and in the study of digital images [1], [7], [8]. The form of clustering introduced in this article has proved to be important in the analysis of brain tissue [9]. The contribution of this paper is an application of Edelsbrunner-Harer nerves as approximating tools in discovering interesting perceptual clusters in Pawlak's painting of landscapes, thus giving us an insight into the style of the artist.

\section{Definitions Plus MNC Construction}

Every Voronoï region of a site $s$ is a convex polygon containing all points that are nearer $s$ than another site in a Voronoï tessellation of a surface. Voronoï regions are strongly near, provided the regions have points in common. In Fig. 1, Voronoï region $\mathrm{N}$ in the tessellation, is the nucleus of a mesh cluster containing all of those polygons adjacent to N. This form of clustering leads to the introduction of what are known as nucleus-clusters.

A Voronoï mesh nucleus is any Voronoï region that is the center of a collection of Voronoï regions adjacent to the nucleus. A maximal nucleus cluster is a collection of a maximal number of Voronoï regions that are strongly near the mesh nucleus. Maximal nucleus clusters (MNCs) serve as indica-

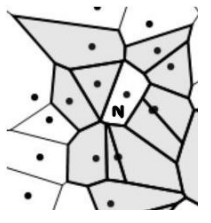

Fig. 1.

Voronoï nucleus tors of high object concentration in a tessellated image.

Definition 1 (Voronoï region $V(s)$ ). Let $E$ be the Euclidean plane, $S \subset E$ (set of mesh generating points), $s \in S$.

$$
V(s)=\{x \in E:\|x-s\| \leq\|x-q\| \text {, for all } q \in S\} .
$$

Nonempty sets $A, B$ in a topological space $X$ equipped with the relation $\stackrel{M}{\delta}$, are strongly near (i.e., strong proximity) (denoted $A \stackrel{\text { M }}{\delta} B$ ), provided the sets have at least one point in common. 
Definition 2. Nucleus Cluster (see, e.g., Fig. 1). Let $X$ be a collection of Voronoï regions containing $N$, endowed with the strong proximity $\stackrel{\mathbb{N}}{\delta}, A \subset X, \operatorname{cl} A=\{x \in X: x \stackrel{\text { M }}{\delta} A\}$ (closure of $A$ ), and

$$
\mathfrak{C} N=\{A \in X: \operatorname{cl} A \stackrel{\text { ᄊ }}{\delta} N\}(\mathrm{NC})
$$

Let $A, B \subset X$ and let $\Phi(x)$ be a feature vector for $x \in X$, a nonempty set of non-abstract points such as picture points. $A \delta_{\Phi} B$ reads $A$ is descriptively near $B$, provided $\Phi(x)=\Phi(y)$ for at least one pair of points, $x \in A, y \in B$ where $\Phi(A)=$ $\left\{\Phi(x) \in \mathbb{R}^{n}: x \in A\right\}$ which are a set of feature vectors

The descriptive strong proximity $\delta_{\Phi}^{M}$ is the descriptive counterpart of $\delta$ defined in the feature space $\Phi(A)$. Let regions $A, B$ be described by a feature vector of the form (x,y,area, diameter $)$. Then $A \delta_{\Phi}^{\text {M }} B$, provided $A$ and $B$ have matching descriptions. Formal proofs of the connection between relations and proximities are given in [1], [4].

Definition 3. Maximum Nuclear Cluster [4]. A nucleus cluster with nucleus $N$ is maximal, provided $N$ has the highest number of adjacent polygons in a tessellated surface denoted by $\max \mathfrak{C} N$. Similarly, a descriptive nucleus cluster is maximal, provided $N$ has the highest number of polygons in a tessellated surface descriptively near $N$, (denoted by $\left.\max \mathfrak{C}_{\Phi} N\right)$.

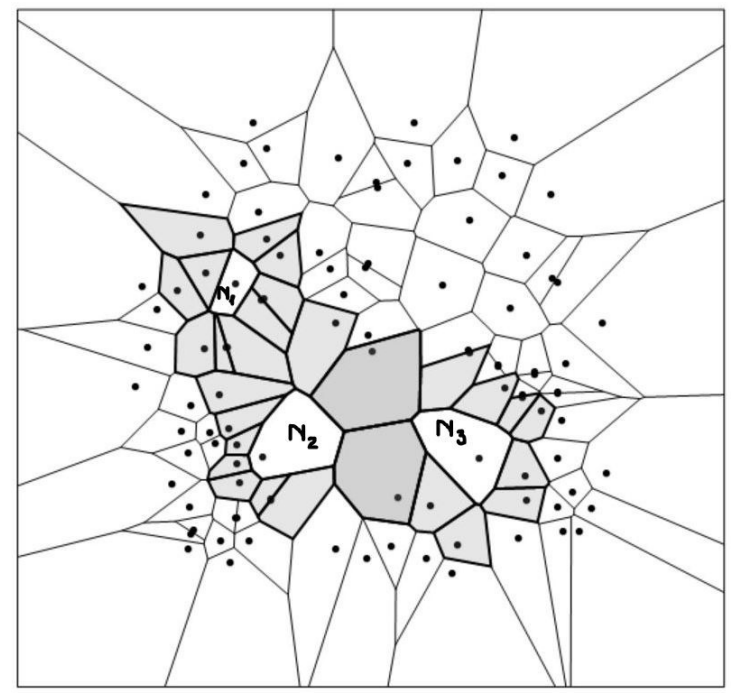

Fig. 2. $\mathfrak{C}_{N_{1}} \stackrel{\wedge}{\delta} \mathfrak{C} N_{2}$ and $\mathfrak{C} N_{1} \delta_{\Phi}^{\stackrel{\wedge}{\mathfrak{n}}} \mathfrak{C} N_{2}$

Example 1. Let $X$ be the collection of Voronoï regions in a tessellation of a subset of the Euclidean plane shown in Fig. 2 with nuclei $N_{1}, N_{2}, N_{3} \in X$. In addition, let $2^{X}$ be the family of all subsets of Voronoï regions in $X$ containing maximal nucleus clusters $\mathfrak{C}_{1}, \mathfrak{C} N_{2}, \mathfrak{C} N_{3} \in 2^{X}$ in the tessellation. Then, for example, int $\mathfrak{C}_{2} \cap \operatorname{int} \mathfrak{C} N_{3} \neq \varnothing$ where int is the interior of a set, since $\mathfrak{C} N_{2}, \mathfrak{C} N_{3}$ share Voronoï regions. Hence, $\mathfrak{C}_{N_{2}} \stackrel{\text { M }}{\delta} \mathfrak{C}_{N_{3}} \neq \varnothing$ (for proof, see [4]). Similarly, $\mathfrak{C} N_{1} \stackrel{\wedge}{\delta} \mathfrak{C} N_{2}$

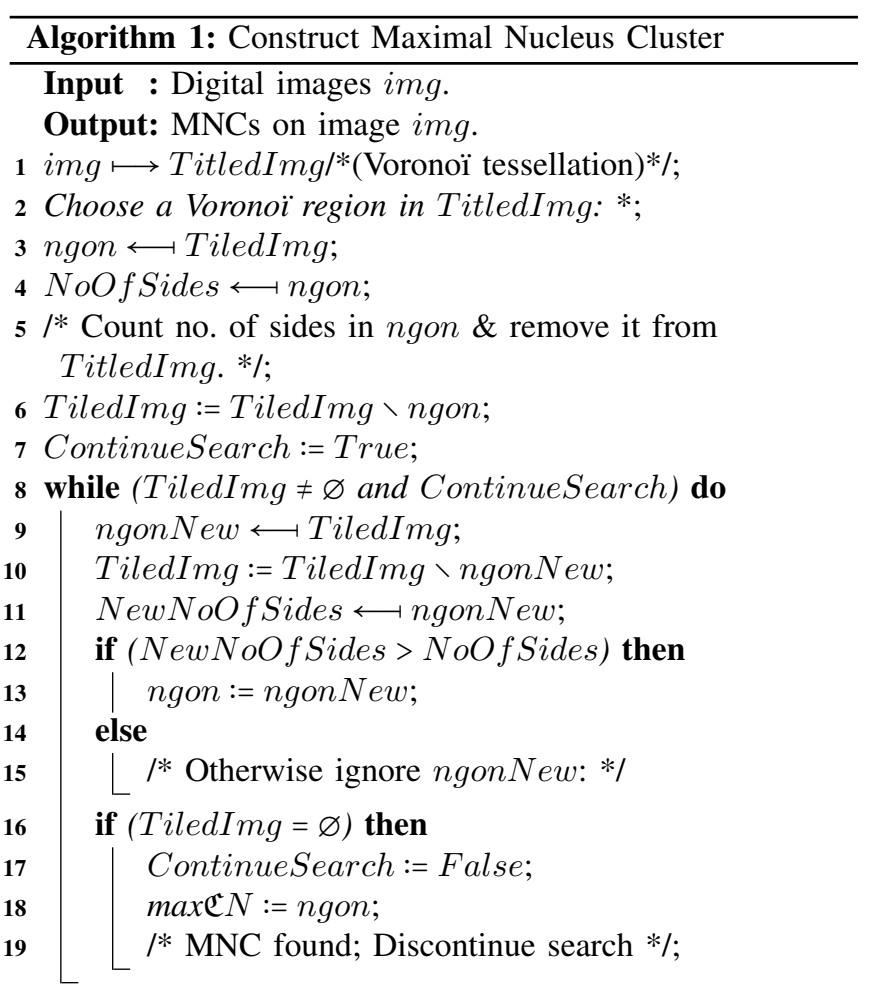

Let $\mathscr{F}$ be a finite collection of sets. An Edelsbrunner-Harer nerve (denoted by $\mathrm{Nrv} \mathscr{F}$ ) consists of all nonempty subcollections of $\mathscr{F}$ that have a non-void common intersection, i.e.,

$$
\operatorname{Nrv} \mathscr{F}=\{X \in \mathscr{F}: \bigcap X \neq \varnothing\} .
$$

Lemma 1. [4] Let $\mathscr{F}_{M N C}$ be a collection of polygons in a Voronoï MNC endowed with the strong proximity $\stackrel{\wedge}{\delta}$. The structure Nrv $\mathscr{F}_{M N C}$ is an Edelsbrunner-Harer nerve.

Theorem 1. [10, §III.2, p. 59] Let $\mathscr{F}$ be a finite collection of closed, convex sets in Euclidean space. Then the nerve of $\mathscr{F}$ and the union of the sets in $\mathscr{F}$ have the same homotopy type.

Theorem 2. [4] Let the nucleus cluster $\mathfrak{C}_{N}$ be a finite collection of closed, convex sets in a Voronoï mesh $V$ in the Euclidean plane. The nerve Nrv $\mathscr{F}_{M N C}$ in $\mathfrak{C} N$ and the union of the sets in $\mathfrak{C} N$ have the same homotopy type.

Theorem 3. [4] Let $X$ be a finite collection of MNC Edelsbrunner-Harer nerves Nrv $\mathscr{F}_{M N C}$ in a Voronoï mesh with nuclei $N$ in the Euclidean plane and let $X$ be equipped with the relator $\left\{\hat{\delta}, \delta_{\Phi}^{\wedge}\right\}$ with strongly close mesh nerves. Each nucleus $N$ has a description $\Phi(N)=$ number of sides of $N$. Then $\bigcap_{\Phi} N r v \mathscr{F}_{M N C} \neq \varnothing$.

\section{EXPERIMENTS AND DISCUSSION}

Let $p\left(x_{1}\right), \ldots, p\left(x_{i}\right), \ldots, p\left(x_{n}\right)$ be the probabilities of a sequence of events $x_{1}, \ldots, x_{i}, \ldots, x_{n}$ and let $\beta \geq 1$. Then the 
Rényi entropy [11] $H_{\beta}(X)$ of a set of event $X$ is defined by

$$
H_{\beta}(X)=\frac{1}{1-\beta} \ln \sum_{i=1}^{n} p^{\beta}\left(x_{i}\right) \text { (Rényi entropy). }
$$

Rényi's entropy is based on the work by R.V.L. Hartley [12] and H. Nyquist [13] on the transmission of information. The information of order $\beta$ contained in the observation of the event $x_{i}$ with respect to the random variable $X$ is defined by $H(X)$. Here, $H(X)$ is used to measure the information levels of maximal nucleus clusters in tesselled paintings by $\mathrm{Z}$. Pawlak (places reflecting the greatest detail by the artist).

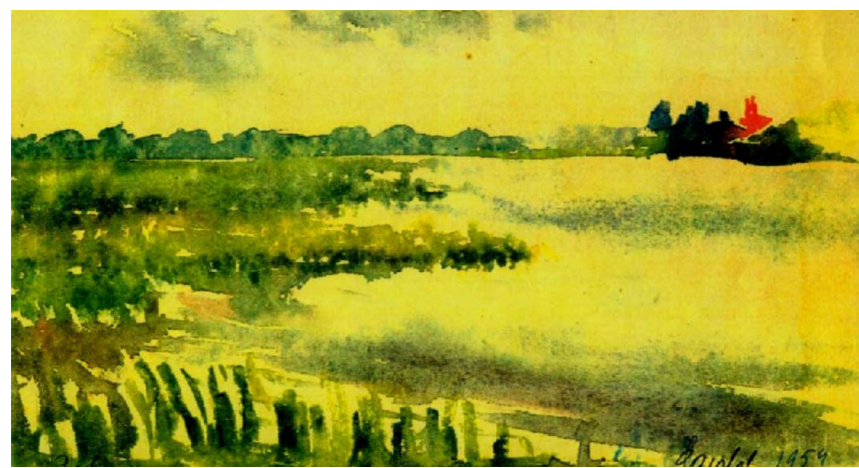

Fig. 3. 1954 Waterscape by Zdzisław Pawlak

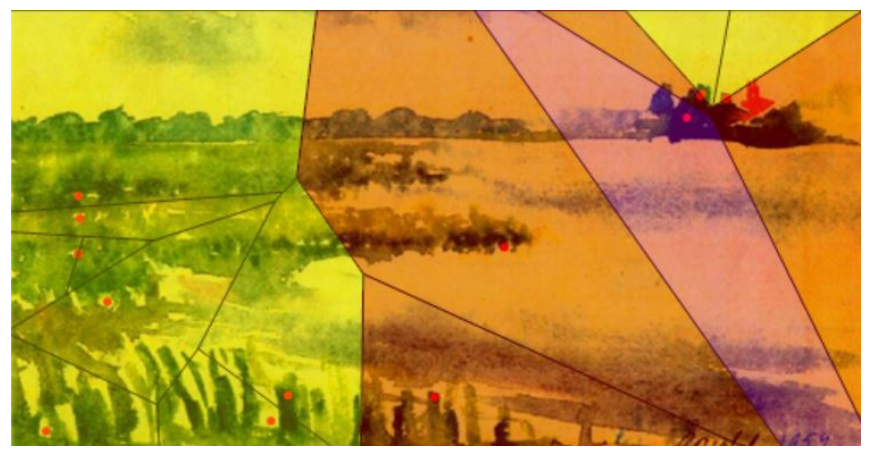

Fig. 4. MNC in 1954 Waterscape by Zdzisław Pawlak

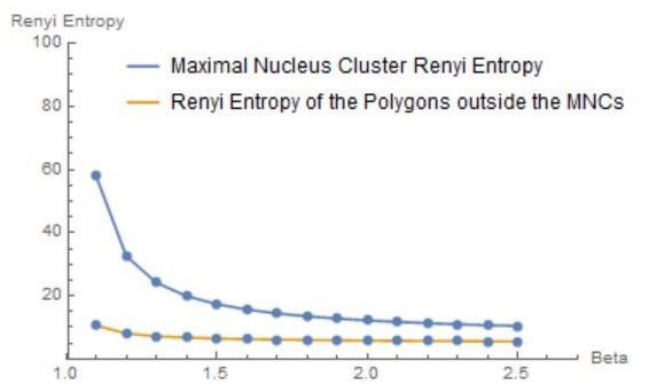

Fig. 5. MNC Rényi's entropy in 1954 Waterscape

The three sample paintings by Z. Pawlak, span 45 years, starting in 1954 and ending in 1999. In Z. Pawlak's paintings, places where the artist rendered with the greatest detail (splashes of colour, slanting brush strokes, clustering of paint

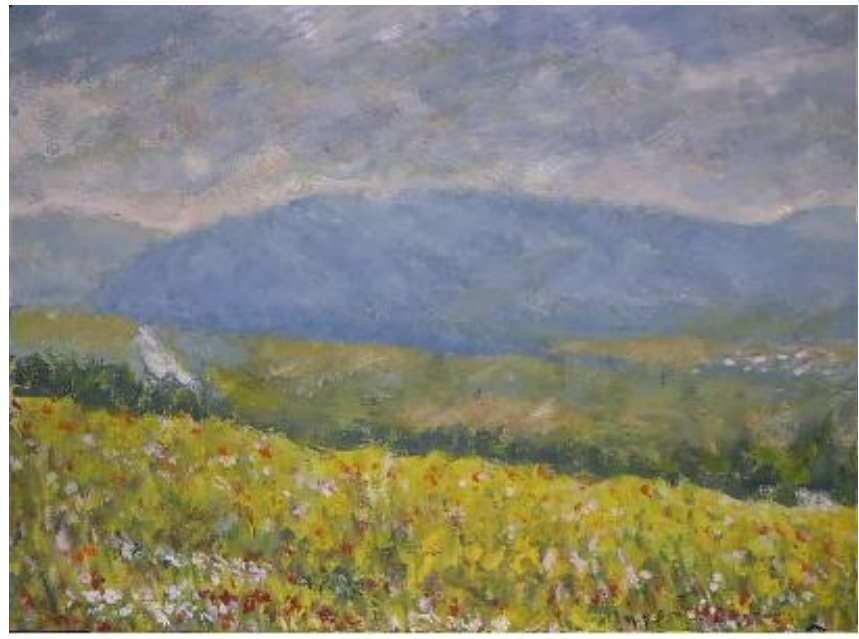

Fig. 6. 1999 Landscape by Zdzisław Pawlak

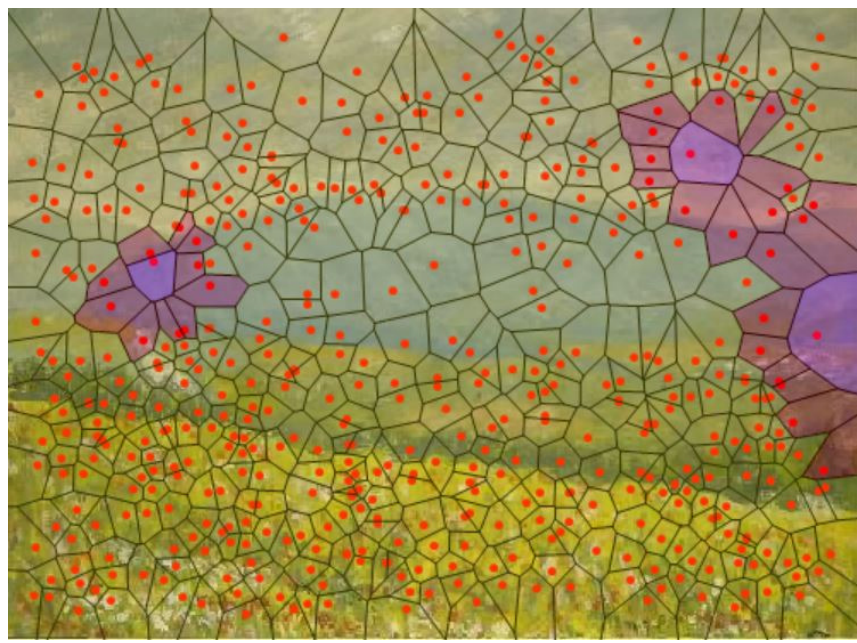

Fig. 7. MNC in 1999 Landscape by Zdzisław Pawlak

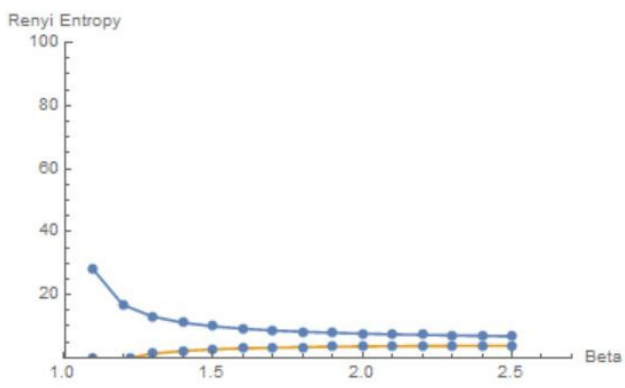

Fig. 8. MNC Rényi’s entropy in 1999 Landscape 


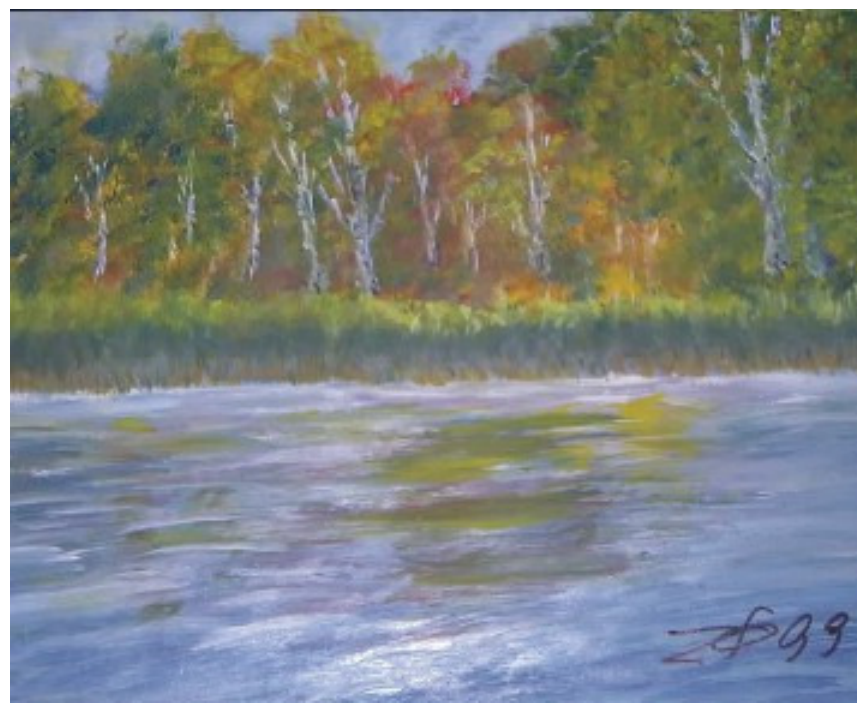

Fig. 9. 1999 Waterscape by Zdzisław Pawlak

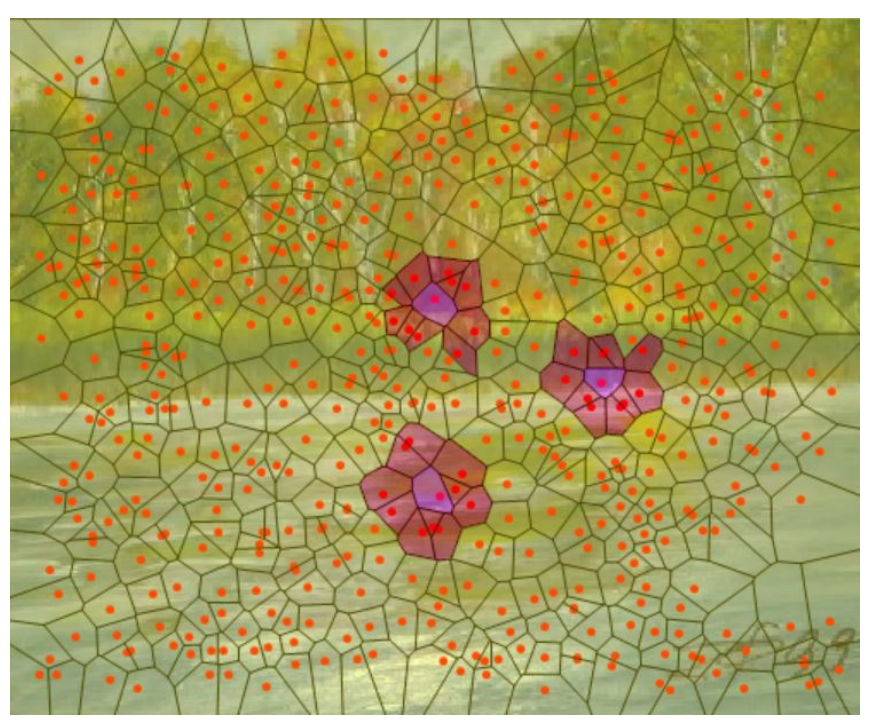

Fig. 10. MNC in 1999 Waterscape by Zdzisław Pawlak

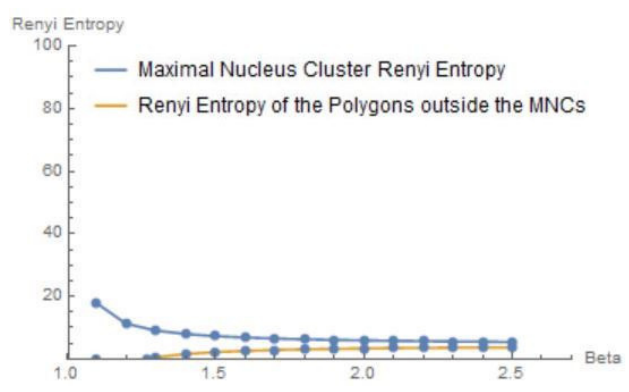

Fig. 11. MNC Rényi's entropy in 1999 Waterscape patches) occurs where the Rényi's entropy is highest. In the examples, the entropy of the MNCs is consistently higher than the non-MNC areas in the paintings. Each of these paintings reflects a style very similar to the impressionist painting by Oscar-Claude Monet (1840-1926). The basic approach was to express one's perceptions of nature in which he created a record of the French countryside. Similarly, Pawlak's perceptions of Polish countryside are represented by dabs of paint to suggest things like buildings (see the red-roofed building in Fig. 3), long and short strokes of colour (see the trees in Fig. 9 and bits of white for distant roads and villages in Fig. 6). The MNCs in Z. Pawlak's paintings occur in those places in the paintings where the artist has expended his greatest efforts on the detailed woodland and waterscape structures he observed.

In conclusion, this paper presents a Voronoï diagram-based clustering which partitions a descriptive space represented by paintings into regions around a set of seed points. The clustering approach leads to the introduction of maximal nucleus clusters that are collections of a maximal number of Voronoï regions that are strongly near the mesh nucleus. It can also be observed that there can be several MNCs depending on the number of selected seed points. It is interesting to note that the Rényi's entropy shown in Figures 5,8,11 indicate the entropy of the MNC is higher than those of the surrounding polygons.

\section{REFERENCES}

[1] J. Peters, Computational Proximity. Excursions in the Topology of Digital Images. Berlin: Springer, 2016, Intelligent Systems Reference Library, 102.

[2] J. Gratus and T. Porter, "Spatial representation: Discrete vs. continuous computational models a spatial view of information," Theoretical Computer Science, vol. 365, no. 3, p. 206.

[3] H. Edelsbrunner, "Modeling with simplical complexes," ser. Proceedings of the Canadian Conference on Computational Geometry, Canada, 1994, pp. $36-44$.

[4] J. Peters and E. İnan, "Strongly proximal edelsbrunner-harer nerves," Proceedings of the Jangjeon Mathematical Society, vol. 19, no. 3, pp. 563-582, 2016.

[5] E. A-iyeh and J. Peters, "Rényi entropy in measuring information levels in Voronï tessellation cells with application in digital image analysis," Theory and Applications of Math. \& Comp. Sci., vol. 6, no. 1, pp. 77-95, 2016.

[6] Q. Du and M. Gunzburger, "Advances in studies and applications of centroidal voronoï tessellations," Numer. Math. Theory Methods Appl., vol. 3, no. 2, pp. 119-142, 2010.

[7] R. Hettiarachchi and J. Peters, "Multi-manifold LLE learning in PatternRecognition,” Pattern Recognition, vol. 48, pp. 2947-2960, 2015.

[8] J. Wang, "Edge-weighted centroidal voronoï tessellation based algorithms for image segmentation," Ph.D. dissertation, Department of Scientific Computing, 2011.

[9] J. Peters, A. Tozzi, and S. Ramanna, "Brain tissue tessellation shows absence of canonical microcircuits," Neuroscience Letters, vol. 626, pp. 99-105, 2016.

[10] H. Edelsbrunner and J. Harer, Computational Topology. An Introduction. Providence, RI: Amer. Math. Soc., 2010, xii+241 pp. ISBN: 978-0-82184925-5, MR2572029.

[11] A. Rényi, "On measures of entropy and information," in Proceedings of the 4th Berkeley Symposium on Math., Statist. and Probability. University of California Press, Berkeley, Calif., 2011, pp. 547-547, vol. 1, Math. Sci. Net. Review MR0132570.

[12] R. Hartley, "Transmission of information," Bell Systems Technical Journal, p. 535, 1928.

[13] H. Nyquist, "Certain factors affecting telegraph speed," Bell Systems Technical Journal, p. 324, 1924. 